\title{
Association of Killer Cell Immunoglobulin-Like Receptor Genes with the Graft versus Host Disease after Related Haematopoietic Stem Cell Transplantation in Patients with Haematological Malignancies from Republic of Macedonia
}

\author{
Aleksandar Petlichkovski ${ }^{1}$, Zlate Stojanoski², Eli Djulejic ${ }^{3}$, Borce Georgievski², Mirko Spiroski ${ }^{1}$
}

${ }^{1}$ Institute of Immunobiology and Human Genetics, Faculty of Medicine, Ss Cyril and Methodius University of Skopje, Skopje, Republic of

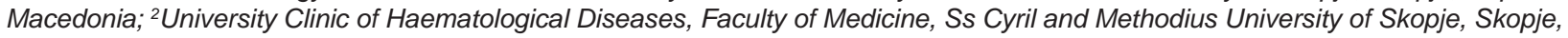
Republic of Macedonia; ${ }^{3}$ Quintiles, Belgrade, Republic of Serbia

\section{Citation: Petlichkovski A, Stojanoski Z, Djulejic E, Georgievski B, Spiroski M. Association of Kille Cell Immunoglobulin-Like Receptor Genes with the Graft versus Host Disease after Related Haematopoietic Stem Cell Transplantation in Haenatopoictic Stem Cell Transplantation in Republic of Macedonia Maced J Med Sci. 2012 Dec 15; 5(4):404-410. http://dx.doi.org/10.3889/ MJMS.1857-5773.2012.0270. \\ Key words: Killer immunoglobulin-like receptor (KIR) gene polymorphism; HLA and KIR genotyping; KIR2DS4; Macedonian patients with HSCT; GVHD. \\ Correspondence: Mirko Spiroski, MD, PhD. Institute of Immunobiology and Human Genetics, Faculty of Medicine, Ss Cyril and Methodius University of Skopje, 1109 Skopje, PO Box 60, Republic of Macedonia. Tel.: +389-2-3110556. Fax: +389-2-3110558. \\ E-mail: mspiroski@yahoo.com \\ Received: 21-Sep-2012; Revised: 15-Nov-2012; Accepted: 07-Dec-2012; Online first:09-Dec-2012 \\ Copyright: ๑ 2012 Petlichkovski A. This is an open-access article distributed under the terms of the Creative Commons Attribution License, which permits unrestricted use, distribution, and reproduction in any medium, provided the original author and source are credited. \\ Competing Interests: The author have declared that no competing interests exist.}

\section{Abstract}

Aim: The aim of this study was to examine the gene frequencies of $16 \mathrm{KIR}$ genes and pseudogenes and $K I R$ genotypes in Macedonian patients with transplanted bone marrow and their sibling donors in treatment of haematological malignancy, and to analyse eventual association of the gene content with the occurrence of a graft versus host disease (GVHD).

Material and Methods: The study was performed on 24 patients and their HLA-matched sibling donors.

Results: Comparison of KIR gene frequencies between the total 24 donors and healthy Macedonians reveals statistically significant difference for KIR2DS1 ( $F=0.481$ in the controls group, and 0.76 in the patients group, $\mathrm{p}=0.004)$. This significance is even higher when the frequency of KIR2DS1 in controls is compared with the frequency in donors from pairs with $\mathrm{GVHD}(\mathrm{F}=0.923, \mathrm{P}=0.002)$. Another significant difference was observed for the frequency of the full-length allele of KIR $2 D S 4 * 001-002$, present in $25.2 \%$ of the control individuals, but in as much as $81.8 \%$ of the recipients of haematopoietic stem cell $(P=0.0005)$. We did not see any statistically significant difference in distribution of the $C 1 / C 1, C 1 / C 2$ and C2/C2 groups among GVHD pairs.

Conclusion: Our results address the difference between the haematopoietic stem cell transplantation settings with sibling and unrelated donors and suggest that the KIR2DS4*001/002 might be a predisposing factor for severe GVHD in sibling HSCT.

\section{Introduction}

Over the last three decades, the transplantation of haematopoietic stem cells coming from bone marrow or peripheral blood from related and unrelated donors has become widely recognized as the only curative treatment for different haematological malignancies and other diseases. Despite of the major advances in histocompatibility testing techniques, more aggressive matching programs, and the developement of more efficient immunosuppressive drugs, this therapeutic procedure is still burdened with a relatively high mortality rate, which ranges between 10 and 30\% [1-4]. As an increasing number of transplants are being performed in 
older patients, and many of them are long term survivors, the incidence of the chronic graft versus host disease (GVHD) rises in the recent years and it reaches rates above $50 \%[5,6]$. Thus, the GVHD becomes one of the main causes of morbidity and mortality associated with haematopoietic stem cells transplantation (HSCT) $[7,8]$.

It is clear by now, that the GVHD involves different effector's cells, several cytokines in abundant quantities and affects the skin, liver and digestive tract but also produces a long-term immune deficiency [9]. However, it should be noted that not all the recipients of even partially unmatched transplants develop clinical disease, while the characteristic exanthema may occur in syngeneic (identical twin) transplants. Among the known factors influencing the outcome of the BMT and development of GVHD, recent studies have indicated that another potential factor influencing the transplantation outcome are the donor-derived natural killer (NK) cells [10-14].

NK cells are bone marrow-derived lymphocytes that mediate early innate immune response to viral infections and transformed malignant cells. Being among the first lymphocyte subsets reconstituting the peripheral blood after allogeneic HSCT [15], NK cells have been attributed several important functions, such as mediation of a graft-versus-leukaemia effect $[16,17]$ promotion of bone marrow engraftment $[1,2]$, and possibly suppression of GVHD $[18,19]$.

The NK cells activity might be activating or inhibiting and it depends on the engagement of the cell surface molecules called killer cell immunoglobulin-like receptors (KIR) with specific class I HLA ligands found on host cells. The KIR molecules may be activating or nonactivating and are encoded by a family of polymorphic and highly homologous genes (14 genes and 2 pseudogenes). Different KIR haplotypes are found in different individuals, and they vary in the number and type of genes present, while the genes KIR3DL3, KIR3DP1, KIR2DL4 and KIR3DL2 are present on virtually all haplotypes and have therefore been termed framework genes [20]. Based on the gene content, many (more then 440 as of November 2012) possible haplotypes have been assigned, and they are grouped into two broad sets, termed A and B [21]. Group A haplotypes contain six inhibitory KIR genes (KIR3DL3, KIR2DL1, KIR2DL3, KIR2DL4, KIR3DL1, and KIR3DL2) and a single activating KIR gene (KIR2DS4) whereas Group B haplotypes contain various combinations of activating and inhibitory KIR genes [22].
Population studies performed over the last two decades have revealed extensive diversity at the KIR gene locus, which derives from both, its polygenic and multi-allelic polymorphism, whereas on the basis of gene content, haplotype B displays a much greater variety of subtypes $[23,24]$.

The aim of this study was to examine the gene frequencies of $16 \mathrm{KIR}$ genes and pseudogenes (KIR2DL1, KIR2DL2, KIR2DL3, KIR2DL4, KIR2DL5, KIR3DL1, KIR3DL2, KIR3DL3, KIR2DS1, KIR2DS2, KIR2DS3, KIR2DS4, KIR2DS5, KIR3DS1, KIR2DP1, and KIR3DP1) and KIR genotypes in Macedonian patients with transplanted bone marrow and their sibling donors in treatment of haematological malignancy, and to analyse eventual association of the gene content with the occurrence of a GVHD. Furthermore, the relationship between KIR genes and the corresponding HLA-C ligands in patients and donors will be analyzed.

To our knowledge, this is the first study of the association of KIR genes with GVHD in transplanted patients with haematological malignancies in the Republic of Macedonia.

\section{Material and Methods}

\section{Population samples}

The study was performed on 24 patients with haematological malignancies treated with transplantation of haematopoietic stem cells between June, 2001 and September 2009 and their related sibling donors for

Table 1: Clinical characteristics of 24 patients with transplanted haemopoietic stem cells from related (sibling) HLA-matched donors.

\begin{tabular}{|c|c|c|c|c|c|c|c|c|}
\hline \multicolumn{2}{|c|}{ Patient } & \multicolumn{2}{|c|}{ GVHD status Diagnosis } & \multirow{2}{*}{$\begin{array}{c}\text { Date } \\
06 / 2001\end{array}$} & \multirow{2}{*}{$\begin{array}{c}\begin{array}{c}\text { Disease } \\
\text { status }\end{array} \\
A D\end{array}$} & \multirow{2}{*}{$\begin{array}{c}\begin{array}{c}\text { Source/ } \\
\text { donor }\end{array} \\
\text { PBSC/f }\end{array}$} & \multirow{2}{*}{$\begin{array}{c}\begin{array}{c}\text { Conditioning } \\
\text { regiment }\end{array} \\
\mathrm{BU}-\mathrm{C} Y+\mathrm{M}\end{array}$} & \multirow{2}{*}{$\begin{array}{c}\text { Months } \\
\text { post-SCT } \\
\mathrm{D}(135+)\end{array}$} \\
\hline$\overline{1.2 v} r(v)$ & V.M. & Chr GVHD & AML & & & & & \\
\hline 2. $\mathrm{D}$ & D.Z. & Chr GVHD & AML & $11 / 2001$ & $A D$ & $\mathrm{PBSC} / \mathrm{f}$ & $\mathrm{BU}-\mathrm{CY}+\mathrm{M}$ & $\mathrm{D}(1290+)$ \\
\hline 3. $\mathrm{P}$ & & Chr GVHD & AML & $1 / 2001$ & $A D$ & $\mathrm{PBSC} / \mathrm{f}$ & $\mathrm{BU}-\mathrm{CY}$ & \\
\hline 4. L & L.S. & & AML & $09 / 2002$ & $\mathrm{CR}$ & $\mathrm{BM} / \mathrm{m}$ & BU-CY & A \\
\hline & B.M. & & AML & $10 / 2002$ & $\mathrm{CR}$ & $\mathrm{PBSC} / \mathrm{m}$ & $\mathrm{FLAG/lda}$ & $\mathrm{D}(660+)$ \\
\hline & J.A. & & CML & $11 / 2002$ & CML bl.tr & $\mathrm{PBSC} / \mathrm{f}$ & $\mathrm{FLAG/lda}$ & $\mathrm{D}(85+)$ \\
\hline 7. A & A.A. & & CML & $03 / 2003$ & CML acc & $\mathrm{PBSC} / \mathrm{f}$ & BU-CY & $D(58+)$ \\
\hline 8. D -10 & D.L.j. & Chr GVHD & CML & $09 / 2003$ & CML acc. & $\mathrm{PBSC} / \mathrm{f}$ & $\mathrm{A} / \mathrm{CY} / \mathrm{Mel}$ & $\mathrm{D}(358+)$ \\
\hline 9. $\mathrm{K}$ & K.N. & Ac. GVHD & $\mathrm{C}$ & $3 / 2004$ & $A D$ & $\mathrm{PBSC} / \mathrm{m}$ & Flumel & $\mathrm{D}(115+)$ \\
\hline 10. $\mathrm{M}$ & M.O. & & Al & $11 / 2004$ & $\mathrm{CR}$ & $\mathrm{PBSC} / \mathrm{m}$ & & A \\
\hline 11. G & Gj.N. & Chr GVHD & CML & $01 / 2005$ & Chr.ph CML & $\mathrm{PBSC} / \mathrm{m}$ & $\mathrm{B}$ & A \\
\hline 12. $T$ & T.O. & Ac. GVHD & $A L L$ & $05 / 2005$ & $A D$ & $\mathrm{PBSC} / \mathrm{m}$ & FLA & $\mathrm{D}(+63)$ \\
\hline 13. $\mathrm{P}$ & P.V. & Ac. GVHD & & $5 / 2005$ & $A D$ & $\mathrm{PBSC} / \mathrm{m}$ & $\mathrm{BU}-\mathrm{CY}+\mathrm{M}$ & $\mathrm{D}(+45)$ \\
\hline 14. D & D.S. & & & 05 & $A D$ & $\mathrm{PBSC} / \mathrm{m}$ & $\mathrm{BU}-\mathrm{CY}+\mathrm{M}$ & $\mathrm{D}(\mathrm{day}+12)$ \\
\hline & & & & & $\mathrm{CR}$ & $\mathrm{PBSC} / \mathrm{f}$ & & $\mathrm{D}(\mathrm{IX/2006)}$ \\
\hline 16. $\mathrm{s}$ & S.D & Ac. GVHD & A & 06 & $\mathrm{CR}$ & $\mathrm{PBSC} / \mathrm{m}$ & BU-CY & $\mathrm{D}$ \\
\hline 17. D & D.B. & & & & & & & $8 / 2$ \\
\hline 18. $\mathrm{S}$ & S.A & $\mathrm{Chr} \mathrm{G}$ & & 37 & & $\mathrm{PBSC} / \mathrm{m}$ & $\mathrm{BU}-\mathrm{CY}$ & A \\
\hline 19. $\mathrm{P}$ & P.M & Chr GVHD & & & CR -2 & $\mathrm{PBSC} / \mathrm{f}$ & & A \\
\hline 20. $\mathrm{H}$ & H.B & & & & $\mathrm{CR}$ & $\mathrm{PBSC} / \mathrm{f}$ & & A \\
\hline 21. $R$ & R.N & & & $12 / 2008$ & $\mathrm{CR}$ & $\mathrm{PBSC} / \mathrm{f}$ & BU & A \\
\hline 22. N & N.M. & & AN & 102 & $C R$ & $\mathrm{PBSC} / \mathrm{m}$ & $\mathrm{BU}$ & A \\
\hline & & & & & & PRSCIf & & \\
\hline & M. & Chr GVHD & & $9 / 20$ & $\mathrm{Cr}$ & $\mathrm{BSC} / \mathrm{m}$ & $\mathrm{BU}-\mathrm{CY}$ & A \\
\hline
\end{tabular}

GVHD, graft versus host disease; $A M L$, acute myeloblastic leukaemia; CML, chronic myeloid leukemia; $A L L$, acute lymphoblastic leukaemia; $C L$, chronic lymphocytic leukaemia; BU, busulfan; $C Y$, acych and G-CSF; Acc, acceleration; BI. Tr. Blast crisis; Chr. Ph., Chronic phase. 
which complete HLA and KIR genotyping data, as well as detailed medical history was available. Distribution according to diagnosis was: acute myeloblastic leukemia 16 (66.7\%), chronic myeloid leukemia 4 (16.7\%), acute lymphoblastic leukemia 3 (12.5\%), and chronic lymphocytic leukemia 1 (4.16\%). As a source of haematopoietic stem-cells, peripheral stem cells or bone marrow aspirates were used. Median number of infused CD34+ cells was: $3.24 \times 10^{6} / \mathrm{kg}$. Twenty patients were treated with myeloablative chemo-therapy regimen busulphan-cyclophosphamide 2, while four of them were transplanted after non-myeloablative regimen, three of which received FLAG-IDA (fludarabine, cytarabine, idarubicin and G-CSF) and one was conditioned using fludarabine-melphalan combination.

During the early post-transplant period, every patient had received $0.2 \mathrm{mg} / \mathrm{kg} \mathrm{b.w}$. intravenous immunoglobulins every week until day +90 . Acute GVHD prophylaxis regimen consisted of Cyclosporine $A$ and Methotrexate (on day $+1,3,6,11$ ) according to Seattle protocol. In the case of cutaneous chronic GVHD, skin biopsies were used for diagnosis. As a first line therapy in cGVHD corticosteroids $1 \mathrm{mg} / \mathrm{kg}$ b.w. and Cyclosporine A $3 \mathrm{mg} / \mathrm{kg}$ b.w.were used, while in case of nonresponders, Mofetyl mycophenolate $1 \mathrm{gr} /$ day, Tacrolimus $0.03 \mathrm{mg} / \mathrm{kg}$ b.w, or Psoralen Ultraviolet light A therapy were introduced. Patients were treated in sterile room, conditioned with HEPA filters, and low microbes diet.

Table 2: KIR gene content in 24 patients and their sibling donors of hematopoietic stem cells, along with their corresponding HLA*C ligands.

\begin{tabular}{|c|c|c|c|c|c|c|c|c|c|c|c|c|c|c|c|c|c|c|c|c|c|}
\hline $\begin{array}{l}\text { Patient and } \\
\text { related donor }\end{array}$ & $\begin{array}{c}K I R \\
3 D L 1\end{array}$ & $\begin{array}{c}K I R \\
2 D L 1\end{array}$ & $\begin{array}{l}K I R \\
2 D L 3 \\
\end{array}$ & $\begin{array}{c}K I R \\
2 D S 4\end{array}$ & $\begin{array}{c}\text { KIR } \\
2 D S 4^{*} 001\end{array}$ & $\begin{array}{c}K I R \\
2 D S 4^{*} 003\end{array}$ & $\begin{array}{c}K I R \\
2 D L 2\end{array}$ & $\begin{array}{c}K I R \\
2 D L 5\end{array}$ & $\begin{array}{c}K I R \\
2 D L 5 A\end{array}$ & $\begin{array}{c}K I R \\
2 D L 5 B\end{array}$ & $\begin{array}{r}K I R \\
3 D S 1 \\
\end{array}$ & $\begin{array}{c}K I R \\
2 D S 1\end{array}$ & $\begin{array}{c}K I R \\
2 D S 2\end{array}$ & $\begin{array}{c}K I R \\
2 D S 3 \\
\end{array}$ & $\begin{array}{c}K I R \\
2 D S 5\end{array}$ & $\begin{array}{c}K I R \\
2 D L 4\end{array}$ & $\begin{array}{c}K I R \\
3 D L 2\end{array}$ & $\begin{array}{c}K I R \\
3 D L 3\end{array}$ & $\begin{array}{c}K I R \\
2 D P 1 \\
\end{array}$ & $\begin{array}{c}K I R \\
3 D P 1\end{array}$ & $\begin{array}{l}\text { HLA*C } \\
\text { group }\end{array}$ \\
\hline V. M. (m) & 0 & 1 & 1 & 1 & 1 & 1 & 0 & 0 & 0 & 0 & 0 & 1 & 1 & 0 & 0 & 1 & 0 & 1 & 1 & 1 & $\mathrm{C} 1 / \mathrm{C} 1$ \\
\hline Sister & 1 & 1 & 1 & 1 & 1 & 1 & 1 & 0 & 0 & 0 & 0 & 1 & 1 & 0 & 0 & 1 & 1 & 1 & 1 & 1 & $\mathrm{C} 1 / \mathrm{C} 1$ \\
\hline D.Z. (m) & 1 & 1 & 1 & 1 & 0 & 1 & 1 & 0 & 0 & 0 & 1 & 1 & 1 & 1 & 1 & 1 & 1 & 1 & 1 & 1 & $\mathrm{C} 1 / \mathrm{C} 2$ \\
\hline Sister & 1 & 1 & 1 & 1 & 0 & 1 & 0 & 0 & 0 & 0 & 1 & 1 & 0 & 0 & 1 & 1 & 1 & 1 & 1 & 1 & $\mathrm{C} 1 / \mathrm{C} 2$ \\
\hline P. E. (f) & 1 & 1 & 1 & 1 & 1 & 0 & 1 & 0 & 0 & 0 & 0 & 1 & 1 & 1 & 0 & 1 & 0 & 1 & 1 & 1 & $\mathrm{C} 1 / \mathrm{C} 1$ \\
\hline Sister & 0 & 1 & 1 & 0 & 0 & 0 & 0 & 1 & 1 & 0 & 0 & 1 & 0 & 0 & 1 & 1 & 1 & 1 & 1 & 1 & $\mathrm{C} 1 / \mathrm{C} 1$ \\
\hline L. S. (m) & 1 & 1 & 0 & 1 & 1 & 1 & 1 & 0 & 0 & 0 & 0 & 1 & 1 & 1 & 0 & 1 & 1 & 1 & 1 & 1 & $\mathrm{C} 1 / \mathrm{C} 2$ \\
\hline Brother & 1 & 1 & 0 & 1 & 1 & 1 & 1 & 1 & 0 & 1 & 0 & 1 & 1 & 1 & 0 & 1 & 1 & 1 & 1 & 1 & $\mathrm{C} 1 / \mathrm{C} 2$ \\
\hline B. M. (f) AA & 1 & 1 & 1 & 1 & 0 & 1 & 0 & 0 & 0 & 0 & 0 & 0 & 0 & 0 & 0 & 1 & 1 & 1 & 1 & 1 & $\mathrm{C} 2 / \mathrm{C} 2$ \\
\hline Brother AA & 1 & 1 & 1 & 1 & 0 & 1 & 0 & 0 & 0 & 0 & 0 & 0 & 0 & 0 & 0 & 1 & 1 & 1 & 1 & 1 & $\mathrm{C} 2 / \mathrm{C} 2$ \\
\hline J.A. (m) & 1 & 1 & 1 & 1 & 1 & 1 & 0 & 0 & 0 & 0 & 0 & 1 & 0 & 0 & 0 & 1 & 1 & 1 & 1 & 1 & $\mathrm{C} 1 / \mathrm{C} 1$ \\
\hline Sister & 1 & 1 & 1 & 0 & 0 & 0 & 0 & 0 & 0 & 0 & 1 & 1 & 0 & 0 & 1 & 1 & 1 & 1 & 1 & 1 & $\mathrm{C} 1 / \mathrm{C} 1$ \\
\hline A. A. (f)AA & 1 & 1 & 1 & 1 & 0 & 1 & 0 & 0 & 0 & 0 & 0 & 0 & 0 & 0 & 0 & 1 & 1 & 1 & 1 & 1 & $\mathrm{C} 1 / \mathrm{C} 2$ \\
\hline Sister AA & 1 & 1 & 1 & 1 & 1 & 0 & 0 & 0 & 0 & 0 & 0 & 0 & 0 & 0 & 0 & 1 & 1 & 1 & 1 & 1 & $\mathrm{C} 1 / \mathrm{C} 2$ \\
\hline D. Lj. (m) & 0 & 1 & 1 & 1 & 1 & 0 & 0 & 1 & 1 & 0 & 1 & 1 & 1 & 1 & 0 & 1 & 1 & 1 & 1 & 1 & $\mathrm{C} 1 / \mathrm{C} 1$ \\
\hline Sister & 0 & 1 & 1 & 1 & 1 & 1 & 0 & 0 & 0 & 0 & 0 & 1 & 1 & 0 & 0 & 1 & 1 & 1 & 1 & 1 & $\mathrm{C} 1 / \mathrm{C} 1$ \\
\hline K. N. (m) & 1 & 1 & 1 & 1 & 1 & 0 & 1 & 1 & 1 & 0 & 1 & 1 & 1 & 0 & 1 & 1 & 1 & 1 & 1 & 1 & $\mathrm{C} 2 / \mathrm{C} 2$ \\
\hline Brother & 1 & 1 & 1 & 1 & 1 & 0 & 1 & 1 & 1 & 0 & 1 & 1 & 1 & 0 & 1 & 1 & 1 & 1 & 1 & 1 & $\mathrm{C} 2 / \mathrm{C} 2$ \\
\hline M. O. (m) AA & 1 & 1 & 1 & 1 & 0 & 1 & 0 & 0 & 0 & 0 & 0 & 0 & 0 & 0 & 0 & 1 & 1 & 1 & 1 & 1 & $\mathrm{C} 1 / \mathrm{C} 1$ \\
\hline Brother AA & 1 & 1 & 1 & 1 & 0 & 1 & 0 & 0 & 0 & 0 & 0 & 0 & 0 & 0 & 0 & 1 & 1 & 1 & 1 & 1 & $\mathrm{C} 1 / \mathrm{C} 1$ \\
\hline Gj. N. (m) & 1 & 1 & 1 & 1 & 1 & 1 & 1 & 0 & 0 & 0 & 0 & 1 & 1 & 1 & 0 & 1 & 1 & 1 & 1 & 1 & $\mathrm{C} 1 / \mathrm{C} 2$ \\
\hline Brother & 1 & 1 & 1 & 1 & 0 & 1 & 1 & 0 & 0 & 0 & 1 & 1 & 1 & 0 & 1 & 1 & 1 & 1 & 1 & 1 & $\mathrm{C} 1 / \mathrm{C} 2$ \\
\hline T. O. (m) AA & 1 & 1 & 1 & 1 & 0 & 1 & 0 & 0 & 0 & 0 & 0 & 0 & 0 & 0 & 0 & 1 & 1 & 1 & 1 & 1 & $\mathrm{C} 1 / \mathrm{C} 2$ \\
\hline Brother & 1 & 1 & 0 & 1 & 0 & 1 & 1 & 1 & 0 & 1 & 0 & 0 & 1 & 1 & 0 & 1 & 1 & 1 & 1 & 1 & $\mathrm{C} 1 / \mathrm{C} 2$ \\
\hline P. V. (f) & 1 & 1 & 1 & 1 & 0 & 1 & 1 & 1 & 0 & 1 & 0 & 1 & 1 & 1 & 0 & 1 & 1 & 1 & 1 & 1 & $\mathrm{C} 1 / \mathrm{C} 1$ \\
\hline Brother & 1 & 1 & 0 & 1 & 0 & 1 & 1 & 1 & 0 & 1 & 0 & 1 & 1 & 1 & 0 & 1 & 1 & 1 & 1 & 1 & $\mathrm{C} 1 / \mathrm{C} 1$ \\
\hline D.S. (m) & 1 & 1 & 1 & 1 & 1 & 1 & 1 & 0 & 0 & 0 & 1 & 1 & 1 & 1 & 0 & 1 & 1 & 1 & 1 & 1 & $\mathrm{C} 1 / \mathrm{C} 1$ \\
\hline Brother & 1 & 1 & 1 & 1 & 0 & 1 & 0 & 0 & 0 & 0 & 0 & 1 & 1 & 1 & 0 & 1 & 1 & 1 & 1 & 1 & $\mathrm{C} 1 / \mathrm{C} 1$ \\
\hline Sh. B. (m) AA & 1 & 1 & 1 & 1 & 0 & 1 & 0 & 0 & 0 & 0 & 0 & 0 & 0 & 0 & 0 & 1 & 1 & 1 & 1 & 1 & $\mathrm{C} 1 / \mathrm{C} 1$ \\
\hline Sister & 1 & 1 & 1 & 1 & 0 & 1 & 1 & 0 & 0 & 0 & 0 & 0 & 1 & 0 & 0 & 1 & 1 & 1 & 1 & 1 & $\mathrm{C} 1 / \mathrm{C} 1$ \\
\hline S. D. (f) AA & 1 & 1 & 1 & 1 & 0 & 1 & 0 & 0 & 0 & 0 & 0 & 0 & 0 & 0 & 0 & 1 & 1 & 1 & 1 & 1 & $\mathrm{C} 1 / \mathrm{C} 2$ \\
\hline Brother & 1 & 1 & 1 & 1 & 0 & 1 & 1 & 0 & 0 & 0 & 1 & 1 & 1 & 0 & 1 & 1 & 1 & 1 & 1 & 1 & $\mathrm{C} 1 / \mathrm{C} 2$ \\
\hline D.B. (m) & 1 & 1 & 1 & 1 & 1 & 1 & 1 & 0 & 0 & 0 & 1 & 1 & 1 & 0 & 0 & 1 & 1 & 1 & 1 & 1 & $\mathrm{C} 2 / \mathrm{C} 2$ \\
\hline Sister & 1 & 1 & 1 & 1 & 1 & 1 & 0 & 0 & 0 & 0 & 1 & 1 & 0 & 0 & 1 & 1 & 1 & 1 & 1 & 1 & $\mathrm{C} 2 / \mathrm{C} 2$ \\
\hline S. A. (f) & 1 & 1 & 1 & 1 & 1 & 1 & 0 & 0 & 0 & 0 & 1 & 1 & 0 & 0 & 1 & 1 & 1 & 1 & 1 & 1 & $\mathrm{C} 1 / \mathrm{C} 2$ \\
\hline Brother & 1 & 1 & 1 & 1 & 1 & 1 & 0 & 0 & 0 & 0 & 0 & 1 & 0 & 0 & 0 & 1 & 1 & 1 & 1 & 1 & $\mathrm{C} 1 / \mathrm{C} 2$ \\
\hline P. M. (m) & 1 & 1 & 1 & 1 & 1 & 1 & 1 & 0 & 0 & 0 & 0 & 1 & 1 & 1 & 0 & 1 & 1 & 1 & 1 & 1 & $\mathrm{C} 1 / \mathrm{C} 2$ \\
\hline Sister & 1 & 1 & 1 & 1 & 1 & 1 & 0 & 0 & 0 & 0 & 0 & 1 & 0 & 0 & 0 & 1 & 1 & 1 & 1 & 1 & $\mathrm{C} 1 / \mathrm{C} 2$ \\
\hline H. B. (f) AA & 1 & 1 & 1 & 1 & 0 & 1 & 0 & 0 & 0 & 0 & 0 & 0 & 0 & 0 & 0 & 1 & 1 & 1 & 1 & 1 & $\mathrm{C} 1 / \mathrm{C} 2$ \\
\hline Sister & 1 & 1 & 1 & 1 & 1 & 1 & 0 & 1 & 1 & 0 & 1 & 1 & 0 & 0 & 1 & 1 & 1 & 1 & 1 & 1 & $\mathrm{C} 1 / \mathrm{C} 2$ \\
\hline R.M. (f) & 1 & 1 & 1 & 1 & 1 & 0 & 0 & 0 & 0 & 0 & 0 & 1 & 0 & 0 & 1 & 1 & 1 & 1 & 1 & 1 & $\mathrm{C} 1 / \mathrm{C} 2$ \\
\hline Sister & 1 & 1 & 1 & 1 & 1 & 1 & 1 & 1 & 0 & 1 & 0 & 1 & 1 & 1 & 0 & 1 & 1 & 1 & 1 & 1 & $\mathrm{C} 1 / \mathrm{C} 2$ \\
\hline N. M. $(f)$ & 1 & 1 & 1 & 1 & 1 & 1 & 0 & 0 & 0 & 0 & 1 & 1 & 0 & 0 & 1 & 1 & 1 & 1 & 1 & 1 & $\mathrm{C} 1 / \mathrm{C} 2$ \\
\hline Brother & 1 & 1 & 1 & 1 & 1 & 1 & 0 & 0 & 0 & 0 & 0 & 1 & 0 & 0 & 0 & 1 & 1 & 1 & 1 & 1 & $\mathrm{C} 1 / \mathrm{C} 2$ \\
\hline N.E. $(f)$ & 1 & 1 & 1 & 1 & 1 & 0 & 1 & 1 & 0 & 1 & 0 & 1 & 1 & 0 & 0 & 1 & 1 & 1 & 1 & 1 & $\mathrm{C} 2 / \mathrm{C} 2$ \\
\hline Sister & 1 & 1 & 1 & 1 & 1 & 1 & 1 & 1 & 0 & 1 & 1 & 1 & 1 & 1 & 0 & 1 & 1 & 1 & 1 & 1 & $\mathrm{C} 2 / \mathrm{C} 2$ \\
\hline M.M. (m)AA & 1 & 1 & 1 & 1 & 1 & 1 & 0 & 0 & 0 & 0 & 0 & 0 & 0 & 0 & 0 & 1 & 1 & 1 & 1 & 1 & $\mathrm{C} 2 / \mathrm{C} 2$ \\
\hline Brother & 1 & 1 & 1 & 1 & 0 & 1 & 0 & 1 & 1 & 1 & 1 & 1 & 0 & 0 & 1 & 1 & 1 & 1 & 1 & 1 & $\mathrm{C} 2 / \mathrm{C} 2$ \\
\hline
\end{tabular}


The clinical characteristics of the patients are shown on Table 1.

\section{HLA and KIR genotyping}

After signing of written consent, genomic DNA was extracted from the peripheral blood leukocytes using standard phenol/chloroform procedure, described elsewhere [25], and stored in the Macedonian Human DNA Bank (hDNAMKD) [26] until processing. All patients and donors were HLA-DNA typed for HLA-A, -B, and $C$ loci by high resolution reverse hybridization technique using commercial kits (Invitrogen Corporation, Brown Deer, WI, USA). HLA-DRB1 locus was genotyped using SBT commercial kit (Pharmacia Biotech, Uppsala, Sweden). For KIR genotyping, commercially available PEL-FREEZ KIR genotyping SSP kit (Dynal Biotech, Brown Deer, WI) was used. It is a PCR-based method (using sequence-specific priming approach) designed to detect the presence or absence of 16 KIR genes. The presence of each KIR gene was determined by the presence of a band of DNA of the expected size. All PCRs contained an internal positive control consisting of an additional pair of primers specific for the growth hormone $(\mathrm{GH})$ gene and a negative control. Individuals were determined negative for a particular KIR gene when a band of expected size was absent in the presence of a band for the $\mathrm{GH}$ gene. We have used external quality control consisting of cell lines from Immunogenetics and Histocompatibility Worskshop Conferences and Centre d' Etude du Polymorphisme Humain.

All analyzed samples were assigned as KIR genotype AA (when homozygosity for KIR haplotype A was detected), or as KIR genotype $B x$ when heterozygosity or homozygosity for KIR haplotype $B$ was typed (KIR genotypes AB and BB).

\section{Statistical analysis}

The occurrence of KIR genes in individuals (frequency $=$ F) was obtained by direct counting. All variables (including conditioning regimens, diagnosis, presence of HLA class I disparity, etc.) were analysed using Fisher's two-sided exact test or Pearson's chisquared test using $2 \times 2$ tables. Crude odds ratios (OR) were calculated within $95 \%$ confidence interval.

\section{Results}

\section{KIR gene frequencies}

The KIR gene content for all 24 analyzed patients and donors, as well as their corresponding HLA-C typings is shown on Table 2. The frequencies of the 16 KIR genes (14 genes and 2 pseudogenes) determined in the 24 donors are shown in Table 3 along with their clinical characteristics and the corresponding frequencies of the 214 healthy Macedonian controls [27]. All 16 KIR genes were observed in both groups of the studied population and framework genes (KIR3DL3, KIR3DP1, $K I R 2 D L 4$, and KIR3DL2) were present in all individuals, except two patients in the GVHD group who lacked KIR3DL2.

Comparison of KIR gene frequencies between the total 24 donors and healthy Macedonians reveals statistically significant difference for KIR2DS1 (F=0.481 in the controls group, and 0.76 in the patients group, $p=0.004)$. This significance is even higher when the frequency of KIR2DS1 in controls is compared with the frequency in donors from pairs with $\operatorname{GVHD}(F=0.923, P=$ 0.002) (Table 3). Another significant difference was observed for the frequency of the full-length allele of KIR2DS4, namely *001-002, which was present in $25.2 \%$ of the control individuals, but in as much as $81.8 \%$ of the recipients of HSC $(P=0.0005)$

\section{Genotype frequencies}

If any of the genes 2DL2, 2DL5, 3DS1, 2DS1, 2DS2, 2DS3, or 2DS5 was present; the genotype was considered as B. If none of these were present, genotype is considered as AA. We have not attempted to distinguish between $A B$ and $B B$ genotypes and called any of this $B x$, nor we have numerated the individual Bx genotypes. We have determined 3 AA genotypes in the group of total 24

Table 3: Comparison of KIR gene frequencies between healthy control subjects from Macedonia and donors of HSC.

\begin{tabular}{|c|c|c|c|c|c|c|c|}
\hline KIR Gene & $\begin{array}{c}214 \text { Controls } \\
\text { N (\%) }\end{array}$ & $\begin{array}{l}\text { 24Total } \\
\text { donors } \\
\mathrm{N}(\%)\end{array}$ & $P$ & $\begin{array}{c}13 \text { GVHD } \\
\text { donors } \\
N(\%)\end{array}$ & $P$ & $\begin{array}{c}13 \text { GVHD } \\
\text { recipients } \\
\mathrm{N}(\%)\end{array}$ & $P$ \\
\hline KIR2DL1 & 201 (94) & $24(100)$ & 0.214 & $13(100)$ & 0.360 & $13(100)$ & 0.360 \\
\hline KIR2DL2 & 126 (59) & $11(44)$ & 0.220 & $6(46.2)$ & 0.367 & 7 (53.8) & 0.721 \\
\hline KIR2DL3 & $192(89.7)$ & $22(88)$ & 0.764 & $11(84.6)$ & 0.561 & $13(100)$ & 0.224 \\
\hline KIR 2DL5A & $60(28)$ & $4(16)$ & 0.234 & $3(23.1)$ & 0.698 & $2(15.4)$ & 0.320 \\
\hline KIR2DL5B & $55(25.7)$ & $6(24)$ & 0.920 & $3(23.1)$ & 0.819 & $1(7.7)$ & 0.140 \\
\hline KIR3DL1 & $201(94)$ & $23(92)$ & 0.706 & $11(84.6)$ & 0.190 & $11(84.6)$ & 0.190 \\
\hline KIR3DL2 & $214(100)$ & $24(100)$ & $\&$ & $13(100)$ & $\&$ & $11(84.6)$ & $\&$ \\
\hline KIR3DL3 & 214 (100) & $24(100)$ & \& & $13(100)$ & \& & $13(100)$ & \& \\
\hline KIR2DS1 & $103(48.1)$ & $19(76)$ & 0.004 & $12(92.3)$ & 0.002 & $10(76.9)$ & 0.044 \\
\hline KIR2DS2 & $122(57)$ & $13(52)$ & 0.790 & $7(53.8)$ & 0.823 & 9 (69.2) & 0.386 \\
\hline KIR2DS3 & 77 (36) & $7(28)$ & 0.508 & $2(15.4)$ & 0.130 & 6 (46.2) & 0.460 \\
\hline KIR2DS4 & 201 (94) & $23(92)$ & 0.706 & $12(92.3)$ & 0.814 & $13(100)$ & 0.360 \\
\hline $\begin{array}{l}\text { KIR2DS4* } \\
001-{ }^{*} 002\end{array}$ & $54(25.2)$ & $13(52)$ & 0.003 & $6(46.2)$ & 0.097 & $9(81.8)$ & 0.0005 \\
\hline $\begin{array}{l}\text { KIR2DS4* } \\
003-009\end{array}$ & $184(86)$ & $21(84)$ & 0.838 & $11(84.6)$ & 0.891 & $10(90.9)$ & 0.368 \\
\hline KIR2DL4 & $214(100)$ & $24(100)$ & $\&$ & $13(100)$ & \& & $13(100)$ & $\&$ \\
\hline KIR2DS5 & $64(30)$ & $9(36)$ & 0.444 & $7(53.8)$ & 0.071 & $3(23.1)$ & 0.600 \\
\hline KIR3DS1 & $84(39.2)$ & $9(36)$ & 0.991 & $6(46.2)$ & 0.621 & 5 (38.5) & 0.955 \\
\hline KIR AA & $46(21.5)$ & $3(12.5)$ & 0.301 & 0 & $<0.0001$ & $2(15.4)$ & 0.600 \\
\hline KIR Bx & $168(79.5)$ & $21(87.5)$ & 0.301 & 13 & $<0.0001$ & $11(84.6)$ & 0.600 \\
\hline
\end{tabular}


donors (12.5\%) which was comparable with the frequency of AA genotypes in controls $(21.5 \%, P=0.301)$. On the other hand, in the group of 13 recipients with GVHD, there were 2 individuals displaying AA genotype (15.4\%, $\mathrm{P}=0.600$ ) which makes the absence of individuals carrying AA genotype exclusive for the donors from pairs with GVHD (Table 3).

\section{KIR HLA*C ligands}

We have also divided the GVHD donors according to the $\mathrm{HLA}^{\star} \mathrm{C}$ ligands they carry into three groups: C1/C1, C1/C2 and C2/C2, and also according the content of up to one and two or more activating genes. We did not see any statistically significant difference in distribution of the above groups among GVHD pairs (Table 4).

Table 4: Distributon of $\mathrm{C} 1 / \mathrm{C} 2$ content and number of activating genes among GVHD donors of HSC.

\begin{tabular}{ccccccc}
\hline GVHD & $\begin{array}{c}\text { Patient } \\
\text { C1/C2 }\end{array}$ & $\begin{array}{c}\text { Patient } \\
\text { C1/C1 }\end{array}$ & $\begin{array}{c}\text { Patient } \\
\text { C2/C2 }\end{array}$ & $\begin{array}{c}\text { s Donors } \\
\text { aKIR content }\end{array}$ & $\begin{array}{c}\text { ?2 Donors } \\
\text { aKIR content }\end{array}$ & $\begin{array}{c}\text { Pearson's } \\
\text { P }\end{array}$ \\
\hline yes & 6 & 4 & 3 & 3 & 10 & N.S. \\
no & 5 & 4 & 2 & 5 & 5 & \\
\hline
\end{tabular}

GVHD, graft versus host disease; aKIR, activating KIR gene; P, significance value; N.S., non significant.

\section{Discussion}

We present the KIR genes distribution in Macedonian patients with haematological malignancies who have been therapeutically transplanted with peripheral stem cells from their HLA matched siblings. Being the first cell population reconstituting the peripheral blood of the patient with HSCT, the NK cells have drown a lot attention lately. On a large series of patients with HSCT from HLA matched, unrelated donors, Cooley et al [28] have shown that patients having KIR2DS2 and $K I R 2 D L 2$ together with absence of KIR2DL3 in donors, are associated with significantly better prognosis. Only recently, another research group has confirmed these findings in a group of patients with thalassemia, again transplanted from unrelated, HLA matched donors [29].

Today, it is generally believed that donors containing at most one activating KIR gene should be avoided if possible, because they could more often lead to severe acute GVHD [30]. In our study, we couldn't confirm these findings, since there was not significant difference in the distribution of donors containing $£ 1$ activating genes among transplant pairs with and without GVHD (3 versus 10, respectively). Similar results and lack of association between the KIR content in sibling
HSCT has been also reported previously [31]. There is one important difference when comparing the unrelated or haploidentical and sibling donor transplant procedures and that is in the dose of donor $\mathrm{T}$ cells that are administered at the time of transplantation because unrelated grafts are more aggressively T-cell depleted. Thus, NK cells that are generated early in the posttransplant period and might remain the predominant lymphoid cell for a prolonged period in the unrelated transplants. In contrast, allografts from siblings are generally T-cell replete and it is possible that this difference in the $\mathrm{T}$-cell and NK-cell immune reconstitution might be responsible for the contrasting clinical outcome of KIR-HLA-C mismatch in the two transplant settings. With the sibling transplantation setting, the favourable KIR/HLA disparity should remain (as their KIR and HLA genes are inherited independently) but might be also reduced in comparison with unrelated transplants [32].

In our cohort, we did find significantly higher frequency of KIR2DS4*001/002 (the full length allele) in donors from pairs with GVHD $(P=0.0005)$, and this finding is concordant with the results published by Bao et al. reporting same highly significant increase in $K I R * 2 D S 4-001 / 002$ full length alleles in donors from a series of 75 unrelated HLA matched HSCT pairs.

The current practice recommendations dictate HLA-matched donors in order to minimize the risk of GVHD; however, there are increasing evidence that vulnerability of recipients with HLA-C2/C2 to relapse is increased, especially in a setting with KIR2DS1 positive donors [33] This challenges the clinicians to consider an HLA-C KIR ligand-mismatched donor in order to take advantage of KIR2DS1-mediated NK alloreactivity towards the leukaemia cells, even in the face of a potentially increased risk of GVHD. It is matter of months, when the algorithms for selection of most appropriate donor of haemopoietic stem cells will include KIR gene/ molecules polymorphisms. The pioneer attempts have already proposed so [34].

In conclusion, our results address the difference between the HSCT settings with sibling and unrelated donors and also suggest that the full length KIR2DS4 activating gene might be a predisposing factor for severe GVHD in sibling HSCT. Our next step should be enlargement of the cohort and analysis of the opposed KIR-HLA interaction within the HSCT, that is promotion of a relapse-free survival through the killing of leukaemia cells by the NK KIR mismatched cells. Finally, we hope that these results suggest that combining of KIR and HLA genotyping could help in finding the ideally balanced 
transplant donors and improve the outcome of transplantation.

\section{Acknowledgement}

The authors thank Elena Cvetkovska (Institute of Immunobiology and Human Genetics, Faculty of Medicine, Ss Cyril and Methodius University of Skopje, Skopje, Republic of Macedonia) for isolation of genomic DNA, and taking care of the Macedonian Human DNA Bank (http://www.hdnamkd.org.mk).

\section{References}

1. Ljungman $P$, Bregni $M$, Brune $M$, Cornelissen J, de Witte T, Dini G et al. Allogeneic and autologous transplantation for haematological diseases, solid tumours and immune disorders: current practice in Europe 2009. Bone Marrow Transplant. 2010;45(2):219-34.

2.Ferrá C, Sanz J, de la Cámara R, Sanz G, Bermúdez A, Valcárcel D, Rovira M,

Serrano D, Caballero D, Espigado I, Morgades M, Heras I, Solano C, Duarte R,

Barrenetxea C, Garcí a-Noblejas A, Dí ez-Martin JL, Iriondo A, Carreras E, Sierra

J, Sanz MA, Ribera JM; GETH (Grupo Español de Trasplante Hematopoyético) and PETHEMA (Programa Español de Tratamiento en Hematologí a), Spanish Society of Hematology. Unrelated transplantation for poor-prognosis adult acute lymphoblastic leukemia: long-term outcome analysis and study of the impact of hematopoietic graft source. Biol Blood Marrow Transplant. 2010;16(7):957-66.

3. Hamilton BK, Copelan EA. Concise review: the role of hematopoietic stem cell transplantation in the treatment of acute myeloid leukemia. Stem Cells. 2012;30(8):1581-6.

4. Hamidieh AA, Alimoghaddam K, Jahani M, Bahar B, Mousavi SA, Iravani M, Behfar M, Jalali A, Jalili M, Hamdi A, Ghavamzadeh A. Non-TBI Hematopoietic Stem Cell Transplantation in Pediatric AML Patients: A Single-center Experience. J Pediatr Hematol Oncol. 2012 Oct 4. [Epub ahead of print]

5. Mohty M, Apperley JF. Long-term physiological side effects after allogeneic bone marrow transplantation. Hematology Am Soc Hematol Educ Program. 2010;2010:229-36.

6. Fraser $\mathrm{CJ}$, Baker KS. The management and outcome of chronic graft-versus host disease. $\mathrm{Br} \mathrm{J}$ Haematol. 2007;138:131-145.

7. Baird K, Cooke K, Schultz KR. Chronic graft-versus-host disease (GVHD) in children. Pediatr Clin North Am. 2010;57(1):297-322.

8. Dong L, Wu T, Gao ZY, Zhang MJ, Kan F, Spellman SR, Tan $X Y$, Zhao YL, Wang JB, Lu DP, Miklos D, Petersdorf E, Fernandez-Vina $M$, Lee SJ. The outcomes of family haploidentical hematopoietic stem cell transplantation in hematologic malignancies are not associated with patient age. Biol Blood Marrow Transplant. 2011;17(8):1205-13.

9. Linhares YP, Pavletic S, Gale RP. Chronic GVHD: Where are we? Where do we want to be? Will IMiDs help? Bone Marrow Transplant. 2012 May 14. doi: 10.1038/bmt.2012.76. [Epub ahead of print]

10. Ruggeri L, Capanni M, Casucci M, et al. Role of natural killer cell alloreactivity in HLA-mismatched hematopoietic stem cell transplantation. Blood. 1999;94:333-339.

11. Ruggeri L, Capanni M, Urbani E, et al. Effective-ness of donor natural killer cell alloreactivity in mismatched hematopoietic transplants. Science. 2002;295:2097-2100.

12. Ludajic K, Balavarca Y, Bickeböller H, Rosenmayr A, Fae I, Fischer GF, Kouba M, Pohlreich D, Kalhs P, Greinix HT. KIR genes and KIR ligands affect occurrence of acute GVHD after unrelated, 12/12 HLA matched, hematopoietic stem cell transplantation. Bone Marrow Transplant. 2009;44(2):97-103.

13. Symons HJ, Leffell MS, Rossiter ND, Zahurak M, Jones RJ, Fuchs EJ. Improved survival with inhibitory killer immunoglobulin receptor (KIR) gene mismatches and KIR haplotype B donors after nonmyeloablative, HLAhaploidentical bone marrow transplantation. Biol Blood Marrow Transplant. 2010;16(4):533-42.

14. Pegram HJ, Ritchie DS, Smyth MJ, Wiernik A, Prince HM, Darcy $\mathrm{PK}$, Kershaw $\mathrm{MH}$. Alloreactive natural killer cells in hematopoietic stem cell transplantation. Leuk Res. 2011;35(1):14-21.

15. Inoue $\mathrm{H}$, Yasuda $\mathrm{Y}$, Hattori $\mathrm{K}$, et al. The kinetics of immune reconstitution after cord blood transplantation and selected CD34 stem cell transplantation in children: comparison with bone marrow transplantation. Int J Hematol. 2003;77:399-407.

16. Zeis M, Uharek L, Glass B, et al. Allogeneic NK cells as potent anti-leukemic effector cells after allogeneic bone marrow transplantation in mice. Transplantation. 1995;20:217-251.

17. Hauch M, Gazzola MV, Small T, et al. Anti-leukemia potential of interleukin-2 activated natural killer cells after bone marrow transplantation for chronic myelogenous leukemia. Blood. 1990;7:2250-2262.

18. Bornhauser M, Thiede C, Brendel C, et al. Stable engraftment after megadose blood stem cell transplantation across the HLA barrier: the case for natural killer cells as graftfacilitating cells. Transplantation. 1999;68:87-88.

19. Asai O, Longo DL, Tian Z, et al. Suppression of graft-versus- 
host disease and ampliû cation of graft-versus-tumor effects by activated natural killer cells after allogeneic bone marrow transplantation. J Clin Invest. 1998;101:1835-1842.

20. Gonzalez, A., McErlean, C., Meenagh, A., Shovlin, T., and Middleton, D. Killer cell immunoglobulin-like receptor allele discrimination by high-resolution melting. Hum Immunol. 2009;70(10):858-63.

21. Liu WR, Kim J, Nwankwo C, Ashworth LK, Arm JP. Genomic organization of the human leukocyte immunoglobulin-like receptors within the leukocyte receptor complex on chromosome 19q13.4. Immunogenetics. 2000;51(8-9):659-69.

22. Hsu KC, Chida S, Geraghty DE, Dupont B. The killer cell immunoglobulin-like receptor (KIR) genomic region: geneorder, haplotypes and allelic polymorphism. Immunol Rev. 2002;190:40-52.

23. Uhrberg M, Valiante NM, Shum BP, Shilling HG, LienertWeidenbach K, Corliss B, Tyan D, Lanier LL, Parham P. Human diversity in killer cell inhibitory receptor genes. Immunity. 1997;7(6):753-63.

24. Middleton D, Gonzelez F. The extensive polymorphism of KIR genes. Immunology. 2010;129(1):8-19.

25. Towner P. Purification of DNA. Essential Molecular Biology T. A. Brown. Oxford, Oxford University Press, 1995:47-54.

26. Spiroski M, Arsov T, Petlichkovski A, Strezova A, Trajkov D, Efinska-Mladenovska O, Zaharieva E. Case Study: Macedonian Human DNA Bank (hDNAMKD) as a source for public health Genetics. Health Determinants in the Scope of New Public Health. B. G. Georgieva L. Sofia, Hans Jacobs Company, 2005:33-44.

27. Djulejic E, Petlichkovski A, Trajkov D, Hristomanova S, Middleton D, Spiroski M. Distribution of killer cell immunoglobulinlike receptors in the Macedonian population. Hum Immunol. 2010;71(3):281-8.

28. Cooley S, Trachtenberg E, Bergemann TL, Saeteurn K, Klein J, Le CT, Marsh SG, Guethlein LA, Parham P, Miller JS,
Weisdorf DJ. Donors with group B KIR haplotypes improve relapse-free survival after unrelated hematopoietic cell transplantation for acute myelogenous leukemia. Blood. 2009;113(3):726-32.

29. Littera R, Orrù N, Caocci G, Sanna $M$, Mulargia $M$, Piras E, Vacca A, Giardini C, Orofino MG, Visani G, Bertaina A, Giorgiani G, Locatelli F, Carcassi C, La Nasa G. Interactions between killer immunoglobulin-like receptors and their human leucocyte antigen Class I ligands influence the outcome of unrelated haematopoietic stem cell transplantation for thalassaemia: a novel predictive algorithm. $\mathrm{Br} \mathrm{J}$ Haematol. 2012;156(1):118-28.

30. Moretta L, Locatelli F, Pende D, Marcenaro E, Mingari MC, Moretta A. Killer Ig-like receptor-mediated control of natural killer cell alloreactivity in haploidentical hematopoietic stem cell transplantation. Blood. 2011;117(3):764-71.

31. Bao XJ, Hou LH, Sun AN, Qiu QC, Yuan XN, Chen $\mathrm{MH}, \mathrm{Chen}$ ZX, He J. The impact of KIR2DS4 alleles and the expression of KIR in the development of acute GVHD after unrelated allogeneic hematopoietic SCT. Bone Marrow Transplant. 2010;45(9):1435-41.

32. Woolfrey A, Klein JP, Haagenson M, et al. HLA-C antigen mismatch is associated with worse outcome in unrelated donor peripheral blood stem cell transplantation. Biol Blood Marrow Transplant. 2011;17:885-92.

33. Cook MA, Milligan DW, Fegan CD, Darbyshire PJ, Mahendra P, Craddock CF, Moss PA, Briggs DC. The impact of donor KIR and patient HLA-C genotypes on outcome following HLA-identical sibling hematopoietic stem cell transplantation for myeloid leukemia. Blood. 2004;103(4):15216.

34. Cooley S, Weisdorf DJ, Guethlein LA, Klein JP, Wang T, Le CT, Marsh SG, Geraghty D, Spellman S, Haagenson MD, Ladner M,Trachtenberg E, Parham P, Miller JS. Donor selection for natural killer cell receptor genes leads to superior survival after unrelated transplantation for acute myelogenous leukemia. Blood. 2010;116(14):2411-9. 